\title{
1,3-Dimethylbenzimidazolinium iodide and 1,3-dimethylbenzimidazoline in reduction processes of $\mathrm{C}=\mathrm{N}$ group of imines
}

\author{
(C) Lidia P. Yunnikova,* and Yulia E. Likhareva \\ Chair of General Chemistry, the Academician D.N. Pryanishnikov Perm State Agrarian-Technological \\ University.Petropavlovskaya St., 23.Perm, 614900.Russia.Email: yunnikova@yahoo.com
}

\begin{abstract}
*Supervising author; ${ }^{+}$Corresponding author
Keywords: 1,3-dimethylbenzimidazolinium iodide, Schiff bases (imines).
\end{abstract}

\begin{abstract}
Interaction between 1,3-dimethylbenzimidazolinium iodide (as an analogue of 1,3-benzodithiolium and 1,3-benzothiolium and tropylium salts), aromatic Schiff bases, and sodium tetrahydroborate in tetrahydrofurane medium (in the presence of imidazole as a cation carrier, or without) at the ratio of starting reagents imine : 1,3-dimethylbenzimidazolinium iodide : sodium tetrahydroborate $=1: 1: 1$ was studied. It was found out that, as basically distinct from the reaction of imines with analogues (1,3-benzodithiolium and 1,3-benzothiolium and tropylium salts) which, while reacting under similar conditions with Schiff bases, form accordingly the products of reductive heterylation $-N$-arylmethyl-4-(1,3-benzodithiol-2-il)aniline, or tropylation $-N$-arylmethyl4-(7-cyclohepta-1,3,5-trienyl)aniline, whereas the reaction of imines with 1,3-dimethylbenz-imidazolinium iodide under similar conditions afforded $\mathrm{N}$-arylmethylanilines in a high yield. This fact enables characterizing the 1,3-dimethylbenzimidazolinium iodide cation as more stable and less electrophilic due to an appreciable delocalization of a positive charge in the cation and, as a consequence, placing it as last (less reactive) in a series of known heteroanalogues (1,3-benzodithiolium $>$ xanthilium $>$ thioxanthilium $>$ tropylium $>N$ methylacridinium > 1,3-dimethylbenzimidazolinium).

The use of 1,3-dimethylbenzimidazoline as a donor of hydride-ion $\mathrm{H}^{-}$(instead of sodium tetrahydroborate) also results in corresponding secondary aromatic amines as the reduction products of Schiff bases.
\end{abstract}

\section{References}

[1] L.P. Yunnikova, T.V. Makhova. Ionic hydroheterylation of $N$-benzylideneanilines in the system sodium tetrahydridoborate-1,3-benzodithiolium salt. Russian Journal of Organic Chemistry. 2006. Vol.42. No.12. P.1864-1865. (russian)

[2] T.A. Akent'eva, and L.P. Yunnikova. Synthesis of amines with tropilidene fragment with anti-fungal potential activity. Butlerov Communications. 2011. Vol.28. No.20. P.80-83. ROI: jbc-02/11-28-20-80

[3] L.P. Yunnikova, T.A. Akentieva, T.V. Makhova. One-pot three-component synthesis of $N$-arylmethyl-4(7-cyclohepta-1,3,5-trienyl)anilines. I. J. Org. Chem. 2013. Vol.3. No.2. P.148-150.

[4] V.G. Harchenko, S.N. Chalaya. Thiopyranes, thiopyrillium salts and related compounds. Saratov University Publishers. 1987.109p. (russian)

[5] I.H. Lee, E.H. Jeoung, M.M. Kzeevoy. J. Am.Chem. Soc. 2001. Vol.123. No.31. P.7492-7496.

[6] W. Doering, L.H. Knox. J. Am. Chem. Soc. 1954. Vol.76. P.3263-3266.

[7] V.A. Izmailsky, E.E. Ivanov, Yu.A. Davydovskaya. Acceptor properties of onium salt as affected by the nature of heteroatom. Russian Journal of Organic Chemistry. 1973. Vol.43. No.11. P.2530-2535. (russian)

[8] L.P. Yunnikova. Interaction of $N$-benzylideneanilines with xanthene. Russian Journal of Organic Chemistry. 1995. Vol.31. No.1. P.76-79. (russian)

[9] L.P. Yunnikova. Reductive heterylation of imines by thioxanthene. Chemistry of Heterocyclic Compounds. 1995. No.7. P.1003-1005. (russian)

[10] RF patent $R U 2132328 C 1$. IPC C07C245/08, 47/58, 47/565. 3-Phenylazo-4-hydroxybenzylideneaniline as activator of alkaline hydrolysis of lignin. Gogotov A.F., Makovskaya T.I., Kanitskaya L.V., Apostolov O.V., Babkin V.A. Applicant for and owner of the patent: Irkutsk Institute of Organic Chemistry of Siberian Branch of the RAS. Application: Nr. 96118046/04 of August 28, 1996, published June 27, 1999.

[11] H.D. Low. J. Chem. Soc. 1912. Vol.101. P.159-165.

[12] K. Brand. Ber. 1909. Vol.42. P.3462 